TABULAR STATISTICS OF THE TWELVE CASES.

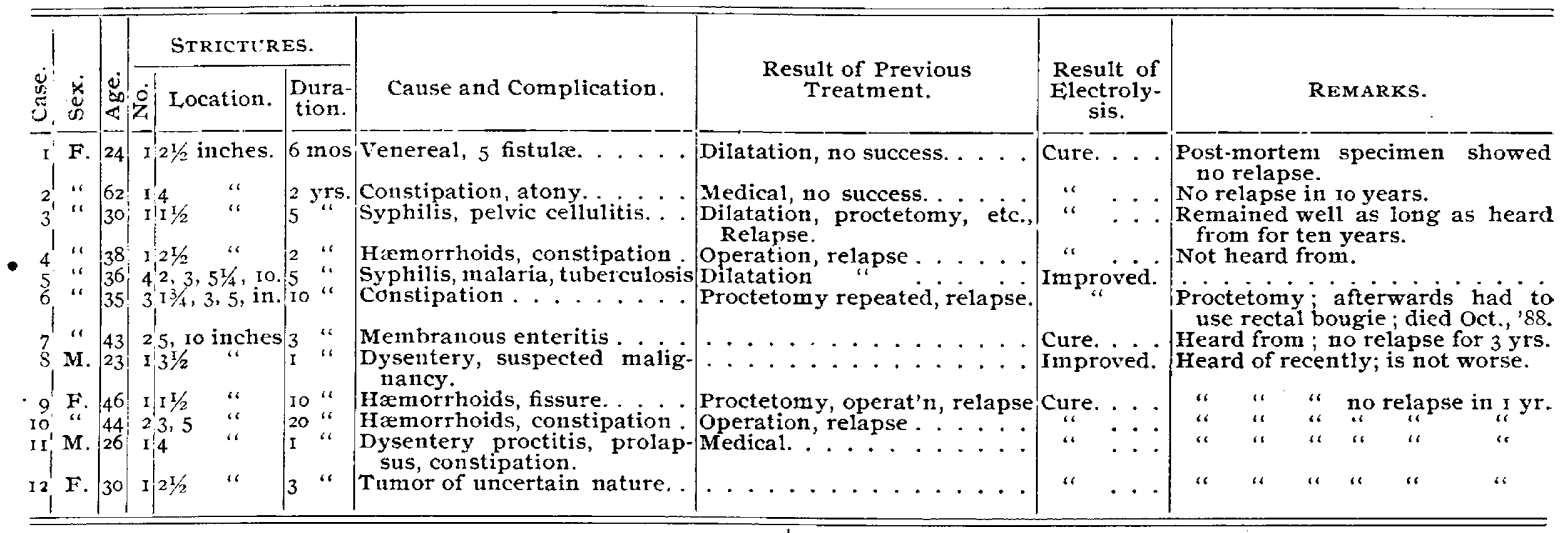

fited; the second was an aggravated case, and the patient too poor to attend to herself, or even to come regularly for treatment. This case was then operated upon, and she had to use a rectal bougie regularly, by which means she kept the stricture from closing up again; but after four years had a relapse with complications and finally died. The improvement in the third case, No. 8 , has been graciously acknowledged by several surgical authorities; however, the patient had to leave the city, thereby interrupting the treatment, and a papillamatous growth, which by some was considered cancerous, complicated the case to such a degree that a cure could scarcely be expected under any treatment. These cases are given just as they were, without claiming any success. The remaining nine cases, however, were cured by the electrolytic treatment, and as far as known no relapse had taken place, which were from one to ten years respectively ; except one case in which nothing has been heard from.

The best results were achieved from the same method as used in treatment of urethral strictures by electrolysis, that means by metal bulbs, as negative, weak currents in intervals. But it is in the nature of the parts treated upon, that the current can be applied stronger and oftener than in the urethra. While in the urethra, a current of five milliamperes is strong, we may increase in the rectum the current to fifteen and sometimes to twenty milliampères, we may prolong a séance from ten to thirty minutes, and repeat it in four days. Stronger currents and the treatment by needles have not proven as successful in my hands.

I hope not to be called too sanguine or an enthusiast, when I come to the following conclusions :

I. Electrolysis in the treatment of stricture of the rectum is not a panacea, on the contrary failures may happen; and probably will ultimately fail if the stricture is due to carcinoma.

2. Electrolysis will give improvement to the rectal stricture, when all other means have failed.
3. Electrolysis will cure a certain percentage of cases, without relapse, better than other modes of treatment, and without the necessity of an after-treatment or using bougies.

4. The best chances for a cure are with the fibrous inflammatory strictures.

5. The best mode of treatment is by a metallic bulb as negative, weak currents and intervals of four days to two weeks. (Further experience in time may change this rule.)

With these conclusions, I recommend electrolysis in the treatment of rectal stricture to the kind consideration of the profession.

68 W. 36th St., New York, June, I889.

\section{THE ORIGIN OF BONE AND JOINT SUPPURATION.}

BEING A DISCUSSION OF THESIS NO. XXIX, "THE MOST FREQUENT CAUSE OF EITHER IS TRAUMATISM," HELD BEFORE THE ST, LOUIS ACADEMY OF MEDICINE.

BY JOSEPH L. BAUER, M.D.,

PROFESSOR OF GENITO-URINARY SURGERY AND ASSOCIATE TO THE CHAIR OF ORTHOP EDIC SURGERY, ST. LOUIS COLLEGE OF PHYSICIANS AND SURGEONS.

Prior to the bacteriological era, the existence of bone and joint disease was ascribed to either scrofula, struma or traumatism. The exponents of the latter based their etiological views, as they do to-day, upon clinical facts and the results of a methodical, yet purely mechanical treatment. The former view owed its prolonged existence to the seeming ridiculousness of ascribing so much responsibility to an injury, ofttimes of apparent harmlessness. Indeed an eminent satisfaction seized them, when the oft repeated question was asked : If as you contend a seeming slight traumatism should produce such dire results, why is it disintegration of bones and joints follows so rarely upon a dislocation or a fracture? This very sophistical question, often determined the views of the majority, and hence the growth of opinion

The Differential Pathology and Diagnosis of Peristitis, Ostitis Osteomyelitis, by Drs. Lous Bauer and $\mathrm{E}$. M. Powers. 
in favor of the traumatic origin of these pathological conditions has been slow. A calm, logical consideration of the question can net but one result. The tendency of all injuries to the body is towards repair, provided the vis medicatrix nature is aided by the skilful assistance of the physician in proper time, and provided: the vis medicatrix nature is not modified in its activity by an inherent or imposed defect. Nor must this inherent or imposed defect be hypothetical, but must be proven by indisputable clinical evidence. Therefore hereditary influences must be establish$e d$ by the positive history of the family, and the imposed influence must be shown, not by the theory of locus minoris resistentice as a selective spot for mycotic elements to germinate and destroy. But, it must be established how these mycotic elements secure entrance into a healthy body, and await an injury to establish their disintegrating properties. And, we may safely assert, that the presence of bacteria or microbes of greater or lesser virulence in the circulating medium of the body, is in itself not sufficient to establish their activity upon a traumatism. In other words, like Darwin's missing link, "there is something wanting" to render such a position irrefutable.

Before discussing the latter proposition, I will have to revert to the simple differences between a traumatised bone and joint and a fracture or a dislocation. In the former the injury is noticeable but a moment, and becomes prominent only when the persistent violation of Nature's laws intensifies the original assault. In the latter, pain and physical defect is serious, and the physician is called at once to relieve it and place it in a position for Nature's reparative process. I will admit that this explanation must perforce be modified, if we take into consideration a few distinctive lesions that follow a badly set fracture and an unreduced dislocation. But, if it modifies the theory of traumatism, it certainly disestablishes the microbic view of locus minoris resistentice.

Prof. Dr. F. Rinne, of the Pathological Department of the University of Greifswald, has just concluded an excellent article in the Archiv. für Klinische Chirurgie, which embodies the detail of a large number of crucial experiments with reference to the subject under discussion, which in my opinion forever sets at rest the theory that there is a locus minoris resistentice for pathogenic microbes circulating in the blood.

Before, however, referring to his theses and the experiments which elucidate them, it may be well to cite an important case, which in a clinical sense adds strength to his position. For if this case shows anything, it demonstrates Mr. Hilton's proposition that, "Rest is the necessary antecedent to the healthy accomplishment of both repair and growth;" or as my friend, Dr. Ber- nays, aptly puts it, "The fact that growth and repair bear an exactly proportionate relation to physiological rest, local as well as general, is certainly the natural suggestion of a means towards an end, which should ever be kept prominently in view by the practitioner of medicine," and he might have added the practitioner of surgery. If the modern view of disease is accepted, the sensible suggestions of both of the gentlemen lose. much, if not all of their weight.

Case $1 .-$ P. S., æt. I I, was sent to me by my esteemed friend, Dr. D. W. McKeen of Russell, Kansas. The little fellow fell off his horse two and a half years previously, and soon thereafter presented a protuberance of the dorsal vertebræ. Before the deformity was noticed, he suffered with girdle pains, weakness of the extremities, gradually lost flesh, appetite grew less, in fact he soon presented the symptoms of spondylitis. When I saw him the deformity had made some headway; the bones and environment were painful to pressure and locomotion, locomotion was impeded by a feeling of weakness in the extremities, emaciation was marked, he had a rise of evening temperature; in fact presented the evidences of decided and rapid decay. The right eye bore evidence of specific disease. All sorts of treatment had been applied to no avail. I did not pay much attention to the condition indicated by the ocular change. Upon my suggestion the mother permitted me to take a cast of the spine, so that Bauer's cuirass, could be properly fitted by it. I also advised her of the necessity of her child maintaining absolute recumbency for at least six months. Hardly a week elapsed, when a change for the better manifested itself in every respect up to within a few weeks before the expiration of four months maintained in the recumbent posture. During all this time his appetite improved, his complexion became clearer, the pains disappeared. At the time mentioned he was seized with a cough, which soon developed a serious pulmonary complication. Another physician was called in who pronounced it miliary tuberculosis, and gave an unfavorable prognosis. This changed condition was communicated to me and my advice sought. I insisted upon his immediate removal to the city, assuming all the responsibility for the long journey. Upon arrival in the city, and after a careful examination, I diagnosed syphilis of the left lung. $\mathrm{My}$ reasons for doing so were first :

I. The history of hereditary syphilis.

2. The absence of irritative symptoms.

3. The rapid and universal involvement of the left lung.

Stuffing with Nicholson's liquid bread, Catlin's beef, wine and iron, claret, milk, cream, etc., and the systematic application of the inunction treatment sufficed to restore the lung to almost complete vitality, and to stimulate $\mathrm{Na}$ - 
ture's forces to renewed activity. When he left for home, the little fellow was to all intents and purposes well, and after a short continuance of the inunction he was given the iodide of potassium in ascending doses. When last heard from, "we cannot give him enough to eat." No symp. toms have developed traceable to the vertebral lesion.

If this case proves anything, it shows that the constitutional vitiation exercised little or no influence upon the spondylitis, but that the enforced rest of the diseased spinal segments was sufficient for the restorative process.

Though our discussion specifically considers osteomyelitis and periostitis, it may not be amiss to include the etiological phases of some joint injuries. For this purpose the following case may be of interest also.

Case 2.-W. J., æt. 22, a lather by trade, whilst trimming a lath with an adze inflicted a perforating wound in the left knee-joint. $\mathrm{He}$ paid little or no attention to the injury, though noting that from the moment of the injury synovial fluid exuded from the opening. He continued to walk about though suffering considerable pain. One week after the injury he called at my office, though hardly able to walk. A careful examination noted loss of contour in the injured knee-joint, pain over the entire joint upon point pressure or movement ; elevated local and general temperature; pus in considerable quantities oozing from the wound. A probe passed into the wound communicated with the joint. I at once decided to place the joint at rest in the extended position and protect the wound by simply applying some absorbent cotton. This was done and the limb immobilized by plaster of Paris bandage. This was at three o'clock in the afternoon; about 8 P. M. I was hastily summoned to see the patient and found that he was delirious and suffering considerable pain, which I attributed to pressure solely. The plaster of Paris shears soon relieved the pressure of the bandage and Bauer's gutter splint was substituted from that time to his complete recovery, a period involving two and a half weeks. No antiseptic medication, either internal or external, was prescribed and yet the patient recovered with full use of his joint.

No one will deny at this day that much has been gained by the acceptance of the microbic origin of suppuration as it occurs in bones and joints. And yet, if we examine the citation of cases, where so-called spontaneous suppurations have ensued in previously healthy subjects, where a general infectious disease or infectious center has not existed heretofore, or at the time of the suppuration, we are forced to doubt the exact force of the modern theory. Thus, Bryck (Langenbeck's Archiv., Bd. xv, "Researches as to Resections") suggests a case of femoral dislo- cation, in which suppuration occurred after repeated attempts at reduction. The patient, I 7 years of age, had previously been healthy.

P. Bruns (Beitrage zur Klin. Chirurgie, Bd. I, S. $237 \mathrm{ff}$. "The Torsion Fractures of the Cylindrical Bones') observed two cases of subcutaneous spiral fractures of the femur suppurate. Both patients died from septicæmia. In one there was also a compound fracture of the tibia with a fissure leading into the knee-joint. The kneejoint was filled with blood and air. Both joint and fracture treated antiseptically. Whilst these injuries were attended in the best of manner, the simple fracture of the femur suppurated. In the pus was found numerous cocci, connected often with diplococci and occuring in the chains, and in fresh preparations manifested a trembling movement.

Steinthal ("Suppuration of Simple Fractures," Deut. Med Wessensch, 1887, No. 21) describes two cases of simple suppurating fractures observed in the clinic at Heidelberg.

The first case a fracture of the femur in a hitherto healthy man, 28 years of age. From the very beginning he had high fever, and four weeks subsequently an abscess developed at the point of fracture, the pus odorless, suppuration of knee-joint, abscess of forearm, thrombosis of femoral vein in opposite limb and decubitus. $\mathrm{Pa}$ tient died. The second case concerned an old luxation of the caput femoris where efforts of reduction produced a fracture. Prior to that time the patient was always well, fourteen days subsequently fever set in and fluctuation appeared, five weeks afterwards an incision discharged one pint of a stinking grayish, green pus, the necrotic head was removed and the patient recovered.

In this case the patient had suffered from diarrhoa, probably from the presence of ingesta due to bad teeth. Steinthal thought that infection proceeded from diarrhœa. The first patient also had a diarrhœa beginning with the tenth day, but his fever commenced at once. S. believed that the infection proceeded through the lungs. Such a supposed infection in the absence of any expressed data where the poison had entered, inasmuch as there was no cutaneous injury, no erysipelas, nor any other manifest complication renders the speculations of S. very questionable.

It may be asserted that it is oftimes impossible to determine a small cutaneous abrasion in a tissue swollen and folded by an injury, but as suppuration is so rare as a consequence of subcutaneous injury, we may leave this point untouched.

If we were to consider the respiratory or digestive tracts as points of entrance into the system and apply this supposition to the cases cited, it is difficult to perceive how the infections material could reach the injured spot, without first affecting the general organism. 
Now, Mr. Chairman, we are acquainted with the experiments of Rosenbach and others that suppuration may ensue as a result of an irritant injected subcutaneously, but as several gentlemen of the Academy do not consider the test a crucial one, I have concluded to present the résumé of Prof. Rinne, as found in his masterly essay and crucial experiments on suppuration and its metastases, and I trust the enthusiasts who look upon the microbes as the essential factor in the suppurative process will peruse it carefully.

"In taking a retrospect of the results of my investigations, we find that a long series of mechanical lesions in which the local presence of pus-cocci existed, has not the power of developing phlegmonous suppurating processes, provided the local injury is protected against the entrance of atmospheric air or oxygen.

"Subcutaneous incisions, even though immediate and concentrated injections of watery mixtures of the staphylococcus aureus and streptococcus were made in the surroundings on the injury, did not suppurate.

"Coccus mixtures were prepared by such concentration of pure cultures, that numberless cocci could be observed in one drop by the microscope. Therefore an uncomplicated, fresh subcutaneous wound is not a fertile soil for pyo-cocci in loco. Resorption is as rapid as in normal tissue, too rapid for the cocci to secure time to develop ptomaines, to irritate the tissues and multiply.

"Glass capsules filled with coccus-mixture and intimately grown into the skin is just as harmless, though the capsules are shattered weeks afterwards, and the cocci are permitted to come in contact with the young cicatricial tissue. Even though we then inject into the swollen late cicatrix pus-microbes in water, they are simply absorbed.

"In old cicatrices also containing particles of glass from rupture of glass capsules, cocci are absorbed without producing suppuration, even with intense infiltration and slower absorption. Cicatricial tissue is therefore not locus minoris resist. entice for the pyo-cocci, on account of equal rapidity of absorption of the injected bacteria, as in late injured tissue; at least absorption is not notably retarded.

"The point of difficulty is to determine the power of resistance in injured tissue or in tissue changed by a cicatrix, towards the absorption of pathogenic pus microbes; the more rapid the resorption the less injurious are the bacteria. The active cellular infiltration, as an expression of the reaction of healthy tissue upon a traumatic injury, as it occurs in an encapsulated foreign body-irritation in the sense of Virchow's cellular pathology-exerts no predisposition for the development of bacteria, but is eminently opposed to it, inasmuch as it is Nature's most effective remedy for the successful combat against inimical parasites.
We can therefore present the following: Subcutaneous injuries of a mechanical nature offer no predilection for the development of pus microbes existent in loco therein when, I, the injury stimulates the absorption activity of the tissues ; 2 , that the mechanical irritation produces a cellular development - two conditions which are to be considered as defensive measures of the healthy organism in the battle of the cells against bacteria (phagocytes, Osler). It follows further, that the tissue lesion, which in this sense produce an inflammatory reaction, is not to be considered as a predisposing factor in the question of metastatic suppuration. But that a mechanical irritant can under certain circumstances produce a predominance of bacterial development, is well demonstrated in the experiments with the woolen threads, ${ }^{2}$ that were saturated with the cocci. Nevertheless, this result in a certain sense emphasizes the previous statement ; for the character of the suppuration evidences, that the resorptive power of the tissues and the active reparative qualities of cells was demonstrated, nevertheless, these pus microbes have had an opportunity of developing pus; and considering some of our other failures, it is to be explained, that the cocci contained in the meshes of the woolen thread occupied a relatively protected position against resorption and the cells, and experience such a rapid multiplication, that ptomaines may develop in the neighboring tissue before the foreign body is completely encapsuled. But the suppuration cannot derelop further, because the active cell proliferation of the surroundings acts as an impenetrable barrier to the onward progress of the bacteria. These succumb gradually and are absorbed, in part by the leucocytes and partly by the pus globules, as shown by the cocci-containing pus globules. The pus corpuscles succumb to fatty degeneration. A mechanical irritation or lesion of the tissues containing pus microbes may suppurate when an irritant foreign body, located in the tissues, gives the cocci an opportunity to develop ptomaines, necessary to the establishment of the suppurative process; that the pus does not take upon itself a phlegmonous character, is explained by the factors shown in the previous experiments, which do not permit the evolution of the pus developing quality of the bacteria to manifest itself; through resorption, the protective cell development and the $a b$ sence of oxygen, a battle, in fact, in which the bacteria always suffer.

"A further possibility for the origin of local suppuration is to be seen in the character of the manifestations after the application of an irritant, as in the impregnated woolen thread. Let us next consider the question of suppuration by chemical agents and its relation to the presence of bacteria. Inasmuch as ptomaines play such an important

2 See experiments iv and v, Archiv, f. Klin. Chirurg., Vol. xxxix, No. 2, I 889, pp. $258,259$. 
part in the suppurative process, and the importance which certain chemical substances, croton oil, ammoniac, have in the development of suppuration, under certain conditions without the aid of the bacteria, but above all, as they can in a definite concentration render the growth of existent bacteria easier in a fibro-mucous exudation, I consider it necessary to make some experiments to ascertain if a migration of microbes from other parts of the body occurred towards abscesses or inflammatory foci produced by chemical agents. I. Inflammatory foci were produced in animals, dogs and cats, by injection of different chemical substances, nitrate of silver, lye, ammoniac, croton oil, cadaverin, etc. Immediately thereafter pure cultures of pus microbes were injected, $(a)$, subcutaneously in remote parts of the body; $(b)$, in the abdominal cavity; $(c)$, in the blood current. In not a single case could these injected pyo-cocci be demonstrated in the chemical abscesses. Even in the development of diffused phlegmonous and ichorous centres with multiple decomposition bacteria of different varieties, could the migration of bacteria be determined in chemical abscesses, inflammation foci, if a separation of both points was sufficient. 2. Even though the experiment was reversed, if phlegmons and abscesses were primarily developed, and then chemical suppuration, the result was negative. 3 . If, as a result of the chemical irritation, a cutaneous necrosis ensued, a migration of atmospheric bacteria frequently ensued, but a suppurative focus never manifested the presence of those cocci which were incorporated subcutaneously through the circulation or into the abdominal cavity. Simultaneously with croton oil, ammonia, cadaverin, pyococci were injected. In a few cases, intense phlegmons coincidental with a partial necrosis of the skin followed. Here both forms of microbes in large numbers were made evident. But more frequently intense cauterization ensued with diffused necrosis and rapid mummification. As a rule, cocci capable of life could not be demonstrated in these experiments. The migration of injected bacteria could not be determined in the inflammatory focus."

Whilst I believe, Mr. Chairman, the results of the experiments made by Dr. Rinne manifestly prove the position taken by thesis 29 , and which I have endeavored to elucidate in the beginning of my remarks, before closing my part of the discussion, I desire to present the following few points which will be of interest: The author says: "From the experiments which I have detailed, I have shown that subcutaneous injuries of tissues with strong lye, nitrate of silver, or any of the other cauterant chemical groups, are not even then a locus minoris resistentice for pyococci, even though these chemicals in themselves produce suppuration when ammoniac or ptomaines are injected (syringe), they can, I, produce abscess when injected in intense concentration; 2, they can prepare the soil when bacteria are injected with them; 3 , they can render the entrance of bacteria possible when they produce necrosis of the skin, but they cannot open the inflammatory centre to the bacteria of the blood."

Let it be distinctly understood, however, that I do not dispute the possibility of concurrent or antecedent infectious diseases, or rather their causal factors derived from the atmosphere, exerting such a continued and persistent change in the general tissue, that they will create a locus minoris resistantice after a traumatism, provided this cell proliferation does not ensue. Nor will I deny the influence which the pneumococcus, gonococcus, streptococcus of rheumatism, erysipelo. coccus, or coccus of diphtheria or typhoid fever may exert upon the general organism and special localities through the agency of the lymph and blood currents, Sonnenschein, Grawitz, Cornil, Bates, E. von Hofman and many others have demonstrated this fact. Latterly Smirnoff, St. Petersburg, I889, has developed this point in his microscopic examinations of the synovial fluid of the knee and shoulder-joint, taken from patients suffering from infectious diseases such as erysipelas, phlegmons, phthisis, typhus, diphtheria and gonorrhoa. But, though the pathogenic elements typical of these diseases were present in the fluid examined, he does not inform us what pathological changes were noted in the articulation from which the synovia was removed.

We must certainly insist that these specific infections must be made out, either by demonstrating their hereditary existence, or development a short period previously, before we can accept the view that these bacteria are responsible for almost all bone and joint suppurations. In order to be scientifically accurate, we must establish the particular sort by irrefutable evidence, and not assume upon theoretical statements that the differences in infective liability are to be sought in this peculiar fitness of the tissues for the reception of microörganisms.

Inasmuch, gentlemen, as our discussion refers particularly to the traumatic origin of periosteal or medullary inflammations, permit me to offer a few remarks upon this point.

Thesis No. I considers bone with its fibrous envelope an anatomical unit. Its histological study confirms this view. Yet, we know by abundant evidence that under certain circumstances, the osteogenetic properties of either may be exerted independently. It follows from this indisputable fact (subperiosteal resections, subperiosteal removal of individual bones), that the views of Macewen are somewhat overdrawn. Yet if we study its clinical phenomena, we are confronted with the interdependence of the bone proper and the periosteum. Thus, it is simply a matter of time, when a periosteal inflammation will be combined with an osteomyelitis, and vice 
versa. But, the early interference of the surgeon can and does interfere with this ultimate connection.

Could I offer a better proof of the traumatic origin of periostitis in a large number of cases than the results of treatment? Bryant has placed in bold relief the infuence of tension on inflammatory affections, and has demonstrated the immediate relief of inflammatory phenomena, after such tension has been removed. We are all acquainted with the prompt results derived from the surgical treatment of both osteomyelitis and periostitis. Subcutaneous division of the inflamed periosteum almost invariably leads to the cessation of the inflammatory process. If this is not done, suppuration with destruction of bone ensues. And what have been the results of treatment in osteomyelitis; of malaria ; typhoid fever ; exposure to damp cold-has not the relief of intra-osseous tension curtailed, even estopped the pathological process ! and will the microbists assert that the well-known virulence and power of tissue penetration of bacteria is prevented by allowing the escape of a few drops of pus or blood?

And how do the microbists explain the origin and recurrence of "The Multiple Recurring Bone Inflammation Common to Mother of Pearl Workers," as described by English (Wiener Med. Presse, I869, Wiener Med. Wochensch., 1870) and Gussenbauer (Archiv. f. Klin. Chirurg., Bd. I8, 1875). Will they accept this pathological condition on the basis of the possibility of "pearl dust" being deposited in the pulmonary parenchyma and, after undergoing some changes, are found as small emboli in the osseous vessels. Or will they accept the assumed, and to me far-fetched, microbic view of Dr. J. Decker (Berl. Klin. Woch., November I I, 1889, p. 975), of Munich?

Permit me to close my remarks with the question: Has it been absolutely proven, that microbes are not scavengers merely?

5I7 Pine Street, St. Louis.

\section{MEDICAL PROGRESS.}

The Therapeutical Application of OxyGEN.-V. VON GyURKOVECHKY. According to this author, inhalations of oxygen are indicated where there is acute or chronic saturation of the blood with carbolic acid; he also recommends its use for imperfect oxygenation in cases of physical weakness, anæmia, leucæmia, chlorosis, indigestion, ennervating nervous diseases, diabetes, hemicrania, sleeplessness, and impotence. The oxygen used must be pure; the quantity to be inhaled depends upon individual peculiarities and the character of the disease, and varies from Io30 litres per day. Old people require less than the young; the enfeebled less than the strong.
The best time for its administration is before breakfast or before the other meals. Accidents have never been observed by the author. Hæmoptysis and aortic aneurism contraindicates its use.-Cent. für Klin. Med.

Labor Complicated by an OVarian Tumor. -In the Gynecological Society of Dresden, MUNCHMEYER reported the case of a multipara 30 years of age, who was admitted to the hospital during labor. An examination revealed a remarkably high position of the head caused by the presence of a large firm tumor which really filled the true pelvis. Attempts at reposition failed; puncture resulted only in the escape of a little fluid blood, the tumor showing no disposition to diminish in size. After death of the child delivery was accomplished by perforating and using the cranioclost, the latter being introduced through an os dilated only to the size of a dollar. For four weeks after labor the tumor, which was connected with the left ovary, remained unchanged, and then began to decrease in size. Ovariotomy was performed and the tumor found to be a spindle-celled sarcoma of the left ovary. The patient made a good recovery and was discharged in three weeks. The points of interest in this case are the extraordinary swelling of the tumor shortly before labor, and the lack of any special symptoms in spite of its malignant. character.-Cent. für Gyñ.

Treatment OF ANTHRAX by HyPODERMic InJections of Carbolic Acid.-DR. DaneT reports four cases of anthrax cured by the hypodermic injection of glyco-phenic acid. The solution was employed in the strength of 3 per cent. of which 4 to 5 cubic centimetres was employed, this amount being injected at several points, and I cubic centimetre being introduced through each puncture.-Journ. de Méd. de Paris.

INSOLUBILITY OF COMPESSED TABLETS. -The case of a boy, æt. 8 years, is reported, in which compressed tablets of antipyrin containing 0.5 grm. each were administered for fever; the tablets passed through the intestinal canal unaltered. In a similar manner compressed tablets. of phenacetin containing the same amount were given to a boy of Io years for neuralgia; these two were discharged undissolved.-Corres. für Schw. Aerzte.

Caffein in Adynamia.-Huchard recommends large subcutaneous injections of caffein as a valuable remedy in adynamic conditions particularly as its action, contrary to that of digitalis, is first upon the nervous system and then upon the heart. He employs from 2.0 to $3.0 \mathrm{grm}$. per day and finds that it can be given with the best results while the benefit of a daily dose of 0.2 to $0.5 \mathrm{grm}$. is illusory. 